\title{
Percutaneous transhepatic biliary drainage and endoscopic retrograde cholangiography in biloma - a rendezvous procedure
}



Fig. 1 Computed tomography performed on the day after endoscopic retrograde cholangiography, showing contrast-enhanced biloma (arrow) and dilated intrahepatic bile ducts (arrowheads).

A 54-year-old man presented with jaundice and an elevated serum bilirubin level of $11.86 \mathrm{mg} / \mathrm{dL}$ after radiofrequency ablation of hepatic metastases from rectal carcinoma. A high-grade stenosis at the common bile duct (CBD) was diagnosed on endoscopic retrograde cholangiography (ERC), and computed tomography showed a 7-cm intrahepatic biloma compressing the CBD ( $\bullet$ Fig. 1 ). A subsequent attempt to bypass the biloma by percutaneous transhepatic biliary drainage (PTBD) failed because the exit of the biloma could not be probed. A pigtail catheter was placed to drain the biloma externally ( $\bullet$ Fig. 2 , - Fig.3).

In a second procedure the patient underwent ERC and PTBD simultaneously. The biloma was successfully probed by ERC with a guide wire, which was caught and extracted with a gooseneck snare via the PTBD access ( $\bullet$ Fig. 4 ) to achieve a pathway through the biloma ( $\bullet$ Fig.5). A pigtail catheter was positioned with its tip lying in the duodenum and its side holes in the bile duct peripheral to the biloma.

The regression of the patient's serum bilirubin concentration to $0.91 \mathrm{mg} / \mathrm{dL}$ and complete occlusion of the biloma 6 months after the procedure showed this treatment strategy to have been successful ( $\bullet$ Fig. 6 ). The principle of healing a bile duct leak is to generate a low-pressure system along the biliary tract by means of internal or external drainage [1 -4]. Sciumè et al. [5] described a technique employing "rendezvous" between ERC and PTBD in highgrade stenosis of bile ducts.

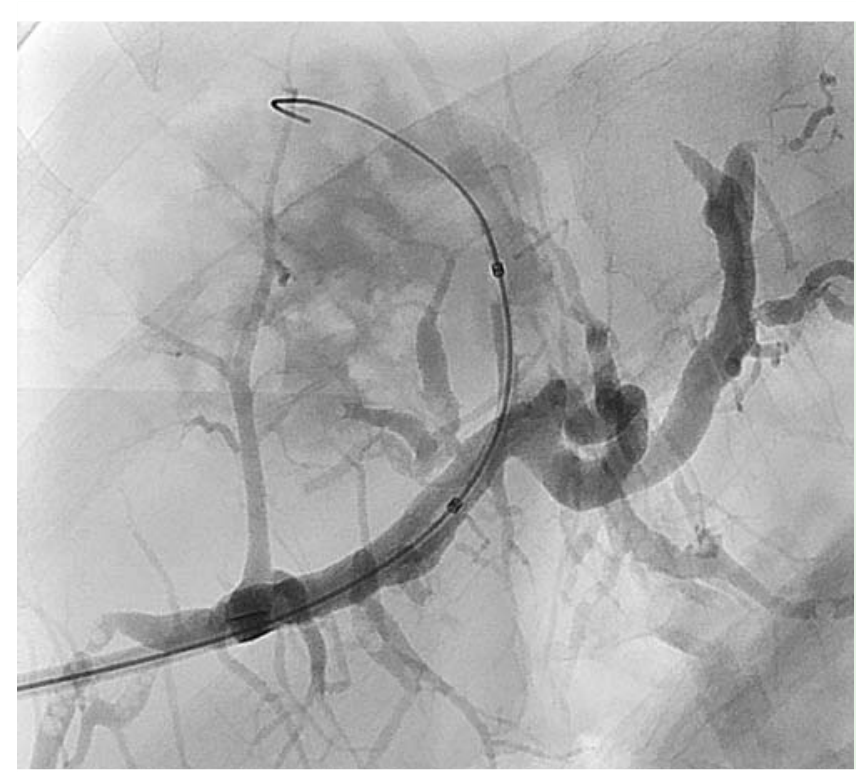

Fig. 2 Percutaneous transhepatic cholangiography guide wire placed in the biloma.

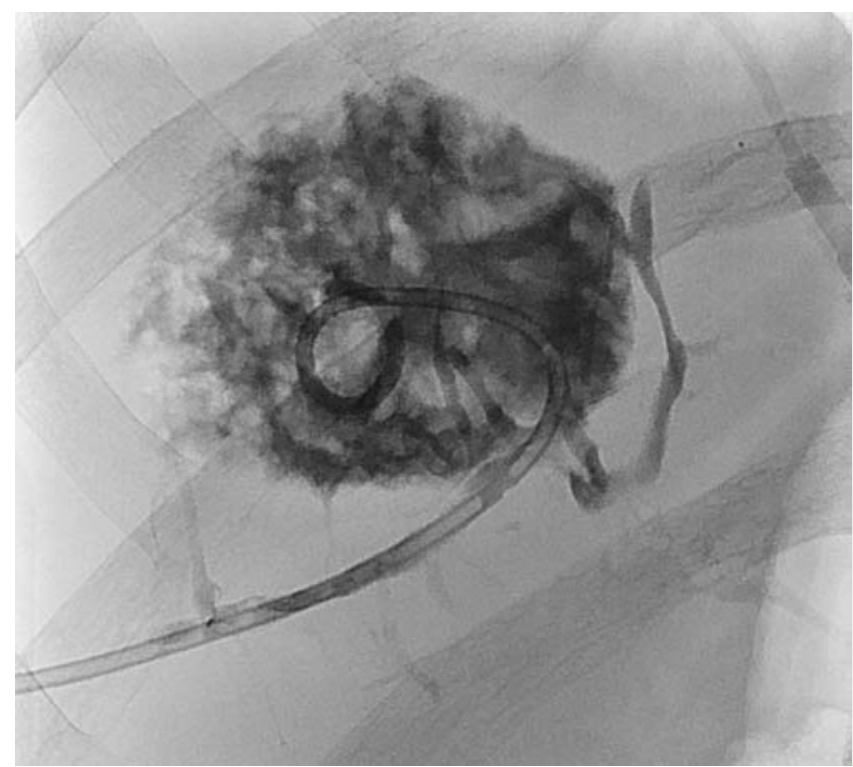

Fig. 3 Biloma drainage with a pigtail catheter placed via the percutaneous transhepatic cholangiography tract.

In our patient, wall necrosis of a central bile duct after radiofrequency ablation led to a valve mechanism that induced formation of a large biloma obstructing the CBD; this in turn led to overpressure, ending in a vicious circle. In this case, it was necessary to puncture the necrotic bile duct accurately and place an internal drain that would bridge the leak and drain the biloma by generating a low-pressure system distal and proximal to the leakage - otherwise the valve mechanism would persist and the leak would remain open. Combining ERC and PTBD in a "rendezvous" technique avoided further surgery.
Endoscopy_UCTN_Code_TTT_1AR_2AJ

Competing interests: None

\section{Bohlsen ${ }^{1}$, P. Kalmar ${ }^{1}$, F. Schreiber ${ }^{2}$, R. H. Portugaller ${ }^{1}$}

${ }^{1}$ Department of Radiology, LKH University Clinic, Medical University Graz, Graz, Austria

2 Department of Gastroenterology and Hepatology, LKH University Clinic, Medical University Graz, Graz, Austria 

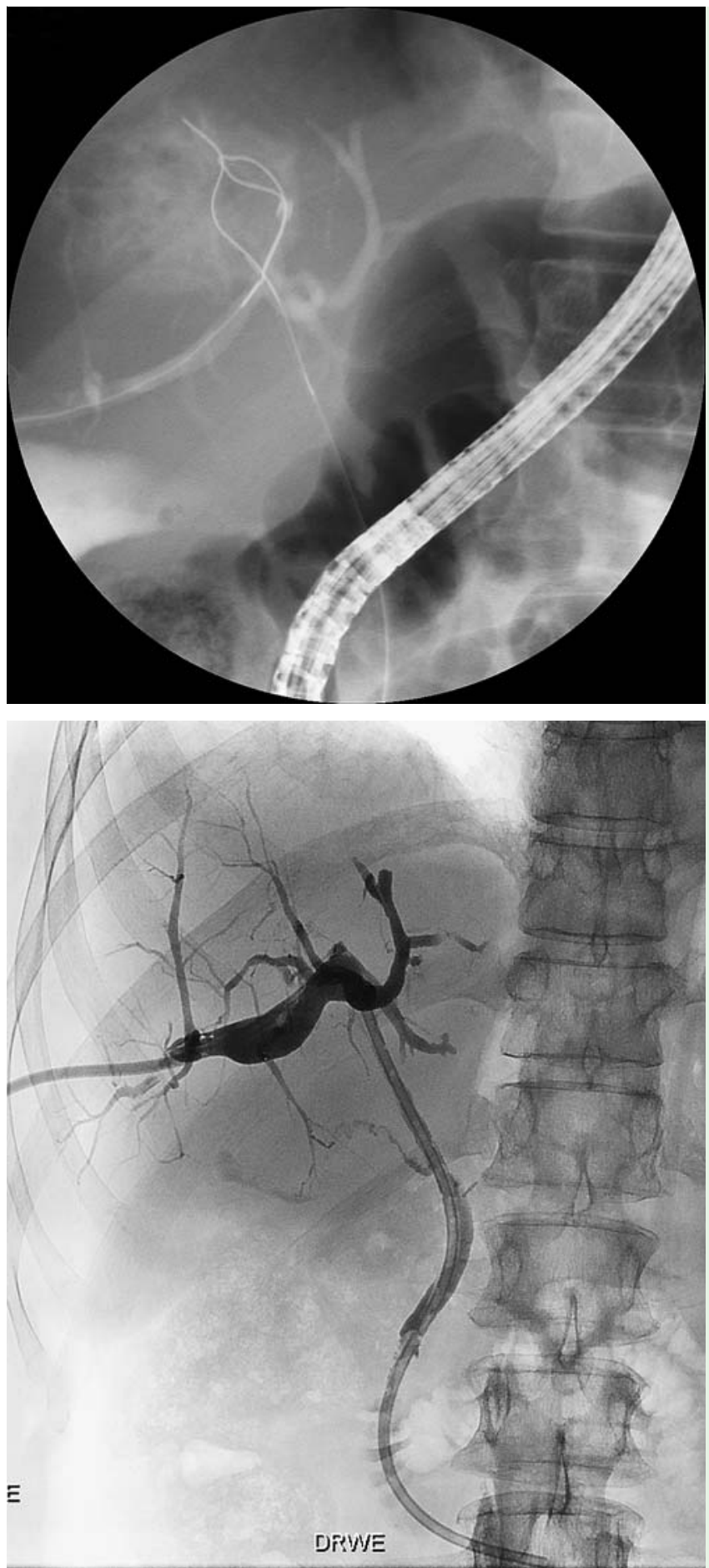

Fig. 4 Rendezvous: the gooseneck snare catches the endoscopic retrograde cholangiography guide wire.

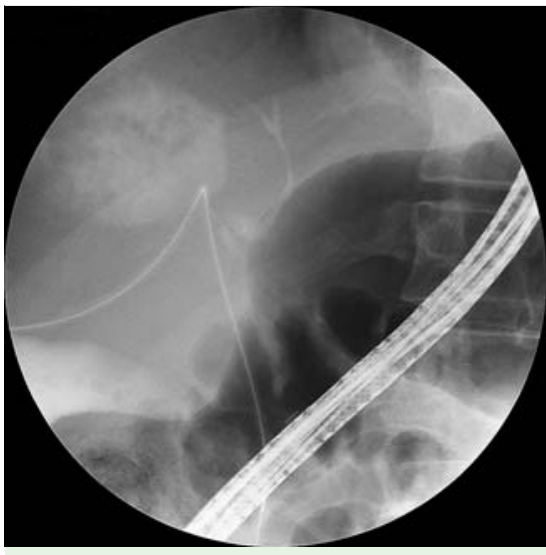

Fig. 5 The pull-through guide wire provides a percutaneous pathway from the peripheral bile duct to the common bile duct and on into the duodenum.

\section{References}

1 Portugaller RH, Hausegger KA. Image-guided interventions. 2. vol. Saunders Elsevier: 2008: 1449-1457

Fig. 6 After temporary placement of an 8.3-Fr pigtail catheter, a 12-Fr Munich percutaneous drainage catheter (Peter Pflugbeil $\mathrm{GmbH}$, Zorneding, Germany) finally crosses the duct necrosis. The bile drains into the duodenum. The external end of the Munich catheter is capped.
2 Vazquez JL, Thorsen MK, Dodds WJ et al. Evaluation and treatment of intraabdominal bilomas. AJR Am J Roentgenol 1985; 144 : 933-938

3 Saab S, Martin P, Soliman GY et al. Endoscopic management of biliary leaks after T-tube removal in liver transplant recipients: nasobiliary drainage versus biliary stenting. Liver Transpl 2000; 6: 627-632

4 Tsukamoto T, Hirohashi K, Osugi $H$ et al. Percutaneous management of bile duct injuries after cholecystectomy. Hepatogastroenterology 2002; 49: 113 - 115

5 Sciumè C, Geraci G, Pisello $F$ et al. "Rendezvous" technique for palliation of neoplastic jaundice: personal experience. Ann Ital Chir 2004; 75: 643-647

\section{Bibliography}

Dol http://dx.doi.org/

10.1055/s-0033-1344573

Endoscopy 2013; 45: E292-E293

(c) Georg Thieme Verlag KG

Stuttgart · New York

ISSN 0013-726X

\section{Corresponding author}

\section{Bohlsen, MD}

Department of Radiology

LKH University Clinic

Medical University Graz

Auenbrugger Platz 9

8036 Graz

Austria

dennis.bohlsen@medunigraz.at 